\title{
BIAS IN INTERPERSONAL PERCEPTION AMONG SOCIOMERIC MUTUAL CHOICES1
}

\author{
Bishwa B. CHATTERJEE and T. K. Hariharan NAIR \\ Balwant Rajput College, Agra, India
}

Bruner and Tagiuri (1954) in their review of the problems of perception of people point out that two distinct trends are recognizable in current research in this area. The first emphasizes accuracy of perception of people or judgment on people; the second focuses on the processes underlying perceiving and judging people. The complex nature of the conceptual and methodological problems associated with the processes of social perception has been critically examined in an important paper by Gage and Cronbach (1955). These authors show convincingly how various artifacts contaminate many reported relationships within processes involving accuracy of interpersonal perception and effective interpersonal relationships, and the need for avoiding such artifacts. On the basis of evidence from available reasearch, both Bruner and Tagiuri, and Gage and Cronbach favor a view that social perception is primarily a 'global' process, and not an analytical, discrete cue-dominated process. While discussing the process of social perception, Gage and Cronbach write, "Then, if the Judge likes the Other, he will predict favorably, socially-acceptable self-descriptions by the Other on a questionnaire or rating scale. If the Other does indeed describe himself favorable, the Judge will be accurate. But the accuracy stems more from a fortuitous concomitance of general favorability sets than from any differentiated perception of the Other." (1955, p.420, italics added).

Let us note that here Gage and Cronbach are limiting themselves to the sources of agreement of a Judge's prediction with the actual self-description of the Other. We propose to extend this line of argument to a somewhat similar situation, where one of the conditions stipulated by Gage and Crobach is fulfilled, viz., the Judge and the Other are favorably disposed towards each other. The Judge, instead of predicting self-description or the Other, may rate the Other on some socially perceivable attributes; the Other, in turn, may be made to rate the Judge on the same attributes. Because or positive bias, as pointed out by Gage and Cronbach, the correlation between the two sets or ratings will tend to be higher when the Judge and the Other are favorably disposed towards each other than when they are not so favorably disposed.

In order to identify persons (Judges and Others) who vary among themselves in the degree of mutually favorable set, we can turn to a sociometric situation. By administering a sociometric test to a group, different types of choice patterns are obtained, such as mutual choices, one-way or unreciprocated choices, indifferences, etc., and it is possible to identify stars, fringers and isolates. It is assumed that a person nominates another person for a sociometric criterion because he feels a certain attraction toward this person, or he expects

1. This paper is based upon a thesis submitted by the junior author, in partial fulfilment for the M.A. degree of the Agra University, 1963. 
to derive a certain satisfaction by having him as a companion. The degree of favorability of disposition, which is assumed to underlie the nomination process, may be conceptualized to vary systematically as follows: it will be greatest among mutual choices (dyadic pairs); it will be somewhat less among one-way choice (unreciprocated choice) pairs; and it will be least among indifferences, that is, non-choosers.

Here, we propose to study this systematic variation in the degree of mutually favorable set and how it is related to the extent of agreement between mutual ratings on a personality type rating scale.

\section{Method}

Design. The response data from the $S$ s were gathered in three successive stages:

1. Administration of the sociometric questionnaire on the group of $S$ s.

2. Obtaining from the $S s$ the names of the most salient qualities or behaviors that they think characterize their nominated friends. A composite rating protocol is framed with the help of these perceived qualities which are most frequently mentioned by the $S$ s.

3. Administration of the rating scale to the $S s$ on an individual basis. This required each $S$ to rate certain members of his group whom he had previously nominated during the sociometric test. The $E$, from an analysis of choice data, decided in advance the names of particular $S$ s that each $S$ would be required to rate. This ensured that each member of a mutual pair rated the other, and that each sociometric star was rated by all those who nominated him, and each star in turn nominated all those that had nominated him. Subjects. Forty-four male students comprizing an entire section of Class $\mathrm{X}$ in a High School in Agra City constituted the group. The Ss knew each other well. Such a large group, which, sociometrically is not very desirable, had to be chosen in order to get an adequate number of mutual pairs and stars.

Sociometric test. A sociometric questionnaire containing three test items was framed for this study. The first item required the $S$ to name five preferred companions for 'sitting near in class'; the second item elicited 5 nominations for 'accompanying to a picnic'; the third item called for 5 nominations 'for staying in a room in a hostel'. There was no stipulation about any preferential ordering among the 5 nominations within the same criterion. The same friend could be nominated for more than one criterion.

Procedure. The junior author (T.K.H.N.) visited the class and made himself familiar with the students to build up a rapport. One week after this initial visit he went to the class and administered the sociometric test. Detailed instruction about the filling up of the questionnaire was given verbally, to supplement the instruction incorporated in the questionnaire.

Qualities for the rating scale. After a brief pause, the junior author visited the class again, and requested the Ss to write down, on separate sheets of paper supplied for this purpose, the names of those qualities that they felt were most prominent in friends nominated by them. They could write the names of as many qualities as they liked. For the nominations made and the qualities mentioned, strict privacy was ensured.

Subjects in general named 3, 4 or 5 qualities as being prominent in their friends. On the basis of the relative frequency of mention of the listed qualities and by merging some similar qualities together, a composite rating scale containing ten items to be rated on a 5-point graphic rating scale was constructed. The five different points along the dimension of most socially approved to least socially approved were marked and short descriptions given to guide the rating of each item of the rating scale.

The ten qualities in the rating scale were: intelligence, truthfulness, politeness, regular attendance, good playing ability, helpfulness, unselfishness, cheerfulness, and good cadetship. Two typical items are shown below:-

1. Good Player

$\begin{array}{llll}\begin{array}{l}\text { Always a good } \\ \text { player }\end{array} & \begin{array}{l}\text { Good player on } \\ \text { most occasions }\end{array} & \begin{array}{l}\text { Sometimes he plays } \\ \text { well and sometimes }\end{array} \\ \text { not } & & 1\end{array}$


7. Helping

$\begin{array}{lllll}\begin{array}{l}\text { He always helps } \\ \text { others }\end{array} & \begin{array}{l}\text { He helps others on } \\ \text { most occasions }\end{array} & \begin{array}{l}\text { Sometimes he helps } \\ \text { others, sometimes } \\ \text { he does not }\end{array} & \begin{array}{l}\text { He does not help } \\ \text { others on most } \\ \text { occasions }\end{array} & \begin{array}{l}\text { He never helps } \\ \text { others }\end{array}\end{array}$

Administration of the rating scale. One week after the administration of the sociometric test, the junior author made individual contact with the $S$ s and obtained their ratings for those of their friends that had been nominated by them. Personal assistance was given to the $S$ s to ensure a uniform procedure in the rating given to their nominations.

The response data were then scored and analyzed for testing the postulated relationships.

\section{RESULTS}

Preliminary analysis of choice data and rating data.

Form the choices exercized by the $S$ s a detailed sociomatrix was prepared. There were about 30 mutual pairs within each of the three criteria, and some of the mutual pairs were repeated over the criteria, i.e., the pair nominated each other for more than one criterion. All mutual choices could very easily be identified by inspection of the matrix of choices.

There were another class of mutual pairs: if one $S$ nominated a companion for one particular criterion, and was in turn nominated by the same companion for a different criterion, we have a dyadic pair, which is called, "between criteria" mutual pair, (Gronlund, 1956). The former mutual pairs are called "within criterion" mutual pairs.

If a person was nominated by $8 S$ s or more, he was called a star. Using this criterion, 20 stars were identified in this group. There were about 50 or so "between criteria" mutual pairs in each of the three "between criteria" pairs: I and II, I and III, and II and III.

Turning to the rating data, two types of scores were obtained from them. Depending upon where the mark is placed for each of the 10 items in the rating scale, scores of 5 through 1 were given, 5 for the most favorable point, 4 for the next one, and so on to 1 , the least favorable. For each rating sheet a total rating score was also obtained by simply summing the separate item rating scores. For agreement analysis, the separate scores on the 10 items were used as a set for each $S$.

Testing of the hypotheses.

Stated in specific terms the following hypotheses are proposed to be tested:

1. The greater the number of nominations an $S$ receives, the more favorably will he tend to be rated by his choosers.

2. Stars will tend to be rated more favorably by persons whom they have also nominated and less favorably by persons who have not also been nominated by the stars themselves.

3. Ratings between mutual choices will tend to be shifted towards the positive end as an increasing function of the number of mentions that underlie the formation of the mutual choices.

4. Agreement between ratings of qualities in the rating scale will be a monotonically increasing function of the number of mentions that underlie the formation of a mutual pair. 
How the favorability of disposition tends to bias the ratings given to the nominees towards the positive end of the rating scale is studied first. Subjects were nominated by $1,2,3$, etc. up to 22 persons. The mean of the total rating scores obtained by each $S$ is first obtained, by dividing the sum of the total rating scores by the number of persons who have given the rating. From the first hypothesis it follows that the mean total rating score will show a systematic increase as the number of persons giving the ratings increases. The mean total rating scores for different number of nominations received are shown in Table 1.

Table 1. Mean Total Rating Scores for Groups of Subjects Receiving Different Number of Nominations.

\begin{tabular}{c|c}
$\begin{array}{c}\text { No. of Nominations } \\
\text { Received }\end{array}$ & $\begin{array}{c}\text { Mean Total Rating } \\
\text { Score }\end{array}$ \\
\hline 1 & 30.0 \\
2 & 33.3 \\
3 & 32.1 \\
4 & 33.8 \\
5 & 37.4 \\
6 & 32.3 \\
7 & 35.1 \\
8 & 36.2 \\
9 & 32.0 \\
10 & 34.2 \\
14 & 41.9 \\
16 & 41.5 \\
22 & 42.6 \\
\hline
\end{tabular}

Inspection of the mean total ratings given in Table 1 shows that they do tend to increase as a function of the number of nominations on which each set of means is based. There are a few reversals, but the over-all trend is unmistakable. The product-moment coefficient of correlation between the number of nominations and the mean total rating score is $.85(\mathrm{df}=11)$, which is significant beyond .01 level. Thus, the first hypothesis appears to be sustainable.

The positive bias operating while rating a friend is further studied by analyzing the ratings related to the sociometric stars. Some of the persons who have rated a star have also been rated by that particular star; and some persons though they have rated the star have not been rated by him, because he has not nominated them. First, the average of the total rating scores given to stars by mutual choosers was computed; next, the average of the rating scores given to stars by non-mutual choosers was found out; and lastly, the average of total rating scores given by stars to their mutual choosers was calculated. These means were calculated separately for each of the three criteria, and then were averaged over the three criteria.

The mean total rating score given by 20 stars to mutual choosers, averaged over the three criteria, was 37.93. This matches rather closely with the mean of the total rating score given to the 20 stars by these mutual choosers, which was 37.50 . However, the mean of total rating scores given to stars by non-mutual choosers was 34.35 , which is in accord with the prediction from hypothesis 2 . The difference between these two means which is equal to 3.15 points was tested by an appropriate $t$ test. The value of $t$ obtained 
was 4.94 with $\mathrm{df}=19$, which is significant beyond .01 level. The second hypothesis is thus found worth retaining with a high degree of confidence. The assumption that the degree of favorability of disposition will tend to be higher among mutual raters and lower among non-mutual raters is found to be confirmed with respect to sociometric stars.

For testing the III and IV hypotheses another independent variable is now taken into consideration: the number of mentions that underlie a particular mutual choice. Let us suppose that person $\mathrm{X}$ has chosen $\mathrm{Y}$ for criterion $\mathrm{I}$. Now, if $\mathrm{Y}$ chooses $\mathrm{X}$ for any one criterion, but one only, then a mutual choice is generated with one underlying mention. If however, $\mathrm{Y}$ chooses $\mathrm{X}$ for two criteria, say Criteria I and II, then a mutual choice has been generated with two underlying mentions. And like this depending upon how $\mathrm{X}$ and Y name each other using one, two or three criteria, we will have mutual choices with underlying mentions of three, four, five and six. The number of mentions that underlie the formation of a set of mutual choices may be assumed to be a determining factor of felt favorability of disposition between mutual pairs. This is proposed to be studied by means of hypotheses III and IV.

All mutual choices were first classified on the basis of the total number of mentions underlying the formation of those mutual pairs. Thus six sets of mutual choices were obtained, from one-mention mutual choices through six-mention mutual choices. For testing Hypothesis III which predicts that the mean mutual rating scores (mean of the total ratings given to each other by the members of a pair) will be an increasing monotonic function of the total number of mentions underlying the mutual choices, the mean rating score (mutual) for each set of mutual choices was separately calculated. The last hypothesis predicts that the degree of agreement between mutual ratings will also be a monotonic increasing function of the total number of mentions that go to the formation of the mutual pairs. For each mutual pair, the separate item scores on the ten items of the rating scale were correlated. The obtained product-moment $r$ values for each set of mutual pairs formed on the basis of total number of mentions were averaged by using Fisher's $z$-transformation. The mean total mutual rating scores and the mean $r$ values, for different sets of mutual pairs formed on the basis of total mentions are shown in Table 2.

Table 2. Mean of Total Rating Scores and of Correlation Coefficients between Separate Mutual Item Scores for Groups of Mutual Choices Formed with Different Number of Total Mentions.

\begin{tabular}{c|ccc}
\hline $\begin{array}{l}\text { Total No. of } \\
\text { Mentions of } \\
\text { Mutual Choices }\end{array}$ & $\begin{array}{c}\text { No. of Mutual } \\
\text { Choice }\end{array}$ & $\begin{array}{l}\text { Mean Total } \\
\text { Rating Score }\end{array}$ & $\begin{array}{l}\text { Mean Mutual } \\
\text { Item-Score } \\
\text { Correlation }\end{array}$ \\
\hline 1 & 14 & 34.6 & .285 \\
2 & 16 & 38.0 & .758 \\
3 & 39 & 36.5 & .825 \\
4 & 10 & 39.9 & .863 \\
6 & 13 & 37.0 & .807 \\
\hline
\end{tabular}

Inspection of the mean mutual total rating scores given in Table 2 inidcates a general tendency towards increase in their value as the number of mentions underlying the set of mutual pairs increases, but this tendency is not very uniform, as there are a few reversals 
in the order of increase. The small number of mutual pairs in certain sets may be responsible for this irregularity. A rank difference coefficient of correlation between the predicted size of the mean mutual total rating score and the actual rank obtained is found to be .44 ( $N=6$ pairs). This is rather low and does not reach significance even at .05 level. The evidence for acceptance or rejection of the III hypothesis thus appears to be equivocal.

The mean correlation coefficients, however, exhibit the predicted monotonic increase more regularly, with only a few reversals. The rank difference coefficient of correlation between the predicted order of the size of the mean $r$ and the actually obtained order of the same is .83 which is significant at .03 level. The size of the correlation is also quite respectable. There, the IV hypothesis about the mutual agreement between separate item scores as a function of the underlying number of mentions for generating the mutual pairs is found to be acceptable.

\section{Discussion}

One assumption which is basic in this study is that it is possible, on the basis of choices made by members of a group under a sociometric situation, and using more than one criterion, to classify the members of the group into categories such that these will vary in the degree of favorability of disposition in a systematic fashion. Thus, in this study using three criteria for making nominations of friends, if some persons are chosen by the same choosers for all the three criteria, the choosers are more favorably disposed towards the chosen than if the same persons are chosen by the same choosers for two criteria, and, they will be still less favorably disposed towards the chosen if the choices are made for only one cirterion. Hence the total number of mentions that underlie a mutual choice will importantly determine the strength of the felt favorable disposition toward the chosen. This favorability set will partly determine how choosers will rate the chosen on a personality type questionnaire or rating scale. An extension of this argument may be made to the situation where we are dealing with measures of agreement between mutual ratings.

There is also another, perhaps less precise, way of ordering the members of the group: on the basis of the number of persons (and not number of mentions) that nominate each member of the group. Here the underlying assumption is that higher the number of persons that choose a member of the group, the greater will be the degree of favorability of disposition toward the chosen.

Gage and Cronbach (1955) had pointed out that the accuracy of interpersonal perception, when indexed by such measures as correlations between self-ratings on some personality traits and ratings on the same traits given by Judges, is contaminated by the positive bias in the ratings given by the Judges to Others, because the Judges are favorably disposed towards the Others. This accuracy of person perception refers only to agreement between ratings given by the Judge and the self-ratings given by the Others. We can, however, extend this argument to the agreement between mutual ratings-ratings which two members of a mutual (dyadic) pair give to each other. Since the formation of a mutual choice pair subsumes a mutually favorable disposition toward each other, the positive bias refered to by Gage and Cronbach may be assumed to enter here, and should show up in an appreciable correlation between mutual ratings. 
The degree of the favorability of mutually felt disposition towards each other in a group can be systematically categorized from high to low if we use appropriate sociometric choice data. Then, a sytematic variation in the extent of agreement between mutual ratings as a function of the degree of mutually favorable disposition can be taken as an evidence of construct validity of the argument presented by Gage and Cronbach with regard to the artifactual source of the accuracy of agreement between ratings.

In the present study, in order to pinpoint this positive bias in mutual ratings, not only agreenents between mutual ratings on a ten-item five-point personality type of rating scale, but also the total rating scores averaged over the number of raters or the number of mentions, were used.

The hypothesis that a person who receives nominations from a large number of persons will receive high mean total rating score, wheras a person who receives nominations from a relatively small number of choosers will receive a low mean total rating score, was found to be acceptable. The systematic increase in the mean total rating score obtained as function of the number of persons nominating, is neatly reflected in a correlation coefficient of $.85(\mathrm{df}=11, \mathrm{p}<.01)$, between number of choosers and mean total rating score.

The II hypothesis predicted that the sociometric stars (arbitrarily defined in this study as those persons who were chosen by 8 or more members of the group) would tend to be rated more favorably by persons whom the stars had also nominated, and would tend to be rated less favorably by persons who had not been nominated by the stars. This hypothesis was also found to be confirmed, there being a statistically significant difference between the mean total ratings given to stars by the chosen and the not-chosen. That the mutuality of the choices made plays an important role in the degree of favorability of disposition between members of a group is the conclusion that follows from this hypothesis. This finding seems to support the view that mutual choices connote greater degree of favorability of disposition than non-mutual or one-way, unreciprocated choices.

The next two hypotheses were framed to study with greter precision the effect of mutuality of choice nominations on the magnitude of the mean rating score and on the extent of agreement between mutual item scores on the composite rating scale.

The assumption basic to these two hypotheses is that the degree of favorability of disposition in mutual choices can be more precisely ordered if we make use of the number of mentions that underlie in the generation of the different mutual pairs, choice date from all the three crietria being used simultaneously. Using this method all mutual pairs identified were classified and placed in one of the six categories: one-mention mutual choices through six-mention mutual choices. It will be seen from Table 2, that the mean of mutual total rating score was 34.61 ( $N=14$ pairs) for one-mention mutual pairs, and it increased to 39.91 ( $N=19$ mutual pairs) for four-mention mutual pairs. The means for remaining categories of total mentions were intermediate in value between these two means. Since the variation in the mean total rating scores does not show any strong systematic trend as predicted by the III hypothesis, the evidence is at best iunclear whether to reject this hypothesis outright or accept it tentatively.

The IV hypothesis predicts that the extent of agreement between the separate item scores between two members of a mutual pair will be a monotonic increasing function 
of the number of mentions that go to generate the mutual pair. The lowest mean correlation between mutual rating scores was $.285(\mathrm{~N}=14)$ and this was for one-mention mutual pairs. The mean correlations, as will be seen in Table 2 , increase more or less regularly as predicted as a function of the number of mentions, reaching the highest value of $.865(\mathrm{~N}=9)$ for six-mention mutual pairs. Thus the last hypothesis is found to be acceptable with a high degree of confidence.

This study seems to confirm the argument that there is enough evidence for a positive bias arising out of the mutually favorable disposition towards each other to be contributing substantially to the reported agreement among ratings which are accepted as a measure of the accuracy of person perception. Three of the four hypotheses postulated here, following from the logic of the presence of positive bias in ratings, are found to be tenable, whereas for the remaining one, the evidence is rather unclear.

It is necessary to point out to one limitation in the design of the present study which can be eliminated in subsequent studies. In this investigation, a person was rated by only those persons who had also nominated him under one or more of the sociometric criteria used. A person was not rated by another person unless he received a nomination from him. By using a larger number of criteria more persons within a group can be made to be nominated, and hence they can be ordered with more precision on the dimension of favorability of disposition. And then, irrespective of whether a person receives nominations from particular persons or not, he could be rated by all the members of the group. With the rating data being more complete, and with the greater degree of precision (actually differentiation) in the favorability of disposition data, more satisfactory and complete tests of the postulated hypotheses will be possible. Until this is done, the evidence from the present study may be of some value in pointing towards the need of further reserach in this complex field.

\section{SUMMARY}

A sociometric questionnaire composed of 3 criteria (sit together, go to a picnic, and live together in a hostel room) was administered to a class of 44 male X grade students of a high school in Agra. Each member of the group also rated those of his classmates whom he had nominated under one or more of the three criteria, on a five-point personality type rating scale containing ten items.

Four hypotheses were formulated relating the degree of favorability of disposition holding between members and rating scores obtained by them. The predictions that followed from these four postulated hypotheses were in general found to be verified. The predictions that the mean total rating score would be systematically related to the number of persons choosing a particular member of the group, and to the total number of mentions that went to generate a set of mutual pairs, were confirmed. Prediction with regard to the extent of agreement between item scores on the rating scale varying as a function of the total number of mentions underlying different sets of mutual pairs was also verified. The evidence about one of the four hypotheses about the postulated relationship between mean total rating scores and the number of mentions underlying the sets of mutual choices was found to be equivocal. A suggestion has been made for further studies which will utilize an improved research design. 


\section{REFERENCES}

Bruner, J. S., and Tagiuri, R. Perception of people. In. G. Lindzey (Ed.) Handbook of social psychology, Vol. II, 635-650. New York: Addison-Wesley. 1954.

Gage, N. L. and Cronbach, L. J. Conceptual and methodological problems in interper- sonal perception. Psychol. Rev., 1955, 62, 411-422.

Gronlund, N. Generality of teachers' sociometric perception: relative judgment accuracy on several sociometric criteria. $J$. educ. Psychol., 1956, 47, 25-31.

MS. received IV 2 '63.

Bishwa Bandhu Chatterjee. (1922-) B. Sc. 1941, M. Sc. 1943, from Banaras Hindu Univ.; B. T. 1951, from Univ. of Gauhati; M. A. 1959, Ph. D. 1960, from Univ. of Illinois. Research Chemist, D. C. M. Chemical Works, Delhi, 1943-46; Lecturer in Chemistry, Union Academy, New Delhi, 1946-52; Lecturer in Education, V.B. Teachers College, Udaipur, 1952-57; Research Assist., Bureau of Educ. Res., Univ. of Illinois, 1957-60; Post Doctoral Instructor, Univ. of Michigan, 1960-61; Prof. of Psychology, Balwant Rajput College, Agra, 1962-63. Research interest: Projective techniques, learning theory, basic education, sociometry.

T. K. Hariharan Nair (1940- ) B. Sc. 1961, Univ. of Kerala; M. A. 1963, Univ. of Agra. Research interests: Industrial psychology, psychology of interpersonal relationships. 